\title{
A THEOREM ON LIE GROUPS
}

\author{
DEANE MONTGOMERY AND LEO ZIPPIN
}

It is the purpose of this note to prove the following theorem:

Theorem 1. Let $G$ be a Lie group $p^{1}$ and $G^{*}$ a compact subgroup of $G$. Then there exists in $G$ an open set $O$ containing $G^{*}$ with the property that for each subgroup $H$ of $G$ lying in $O$ there is an element $g$ of $G$ such that $g^{-1} \mathrm{Hg}$ is in $\mathrm{G}^{*}$.

Roughly, the theorem says that each subgroup near enough to $G^{*}$ can be transformed into $G^{*}$ by an appropriate element of $G$. This result can be regarded as a generalization of the known fact that Lie groups cannot have arbitrarily small subgroups (other than the identity), although it was not from this point of view that our interest arose. To make our meaning clear, assume that $G^{*}$ is an invariant subgroup so that the factor group $G / G^{*}$ is also a Lie group. If there were in $G$ a subgroup $H$ near $G^{*}$ it would go, by the homomorphism taking $G$ into $G / G^{*}$, into a subgroup near the identity of $G / G^{*}$. The only subgroup of $G / G^{*}$ near the identity is the identity itself which means that if $H$ is to be near $G^{*}$ it must actually be a subgroup of $G^{*}$. We see that when $G^{*}$ is an invariant compact subgroup of $G$, the conclusion of the theorem is true in a trivial sense.

Our proof of Theorem 1 in the more general situation is based on certain facts about the way in which $G$ operates on the coset space $G / G^{*}$ which will be denoted by $M$. This is the space whose points are the cosets $g G^{*}$ of $G^{*}$ in $G$. The group $G$ acts transitively on $M$ which can be regarded as a Riemannian space and Cartan ${ }^{2}$ has shown that there exists in $M$ a Riemannian metric for which $G$ is a group of isometries. This fact will be of great importance in what follows.

We begin, as we may, by supposing that $M$ is endowed with a Riemannian metric invariant under $G$ and, furthermore, we assume that $M$ has been made into a metric space (Fréchet) in the usual way

Presented to the Society, September 5, 1941; received by the editors August 29, 1941.

${ }^{1}$ For all definitions and theorems on topological groups used in this paper see Pontrjagin, Topological Groups. The term compact, as used here, implies that the set is closed.

${ }^{2}$ La Thêorie des Groupes Finis et Continus et l'Analysis Situs, Mémorial des Sciences Mathématiques, vol. 42, p. 43. For an excellent summary of properties of isometries and geodesics which will be useful here, see the paper by Myers and Steenrod, The group of isometries of a Riemannian manifold, Annals of Mathematics, (2), vol. 40 (1939), pp. 400-416. 
by letting the distance $d(x, y)$, between two points $x$ and $y$ be the greatest lower bound of the lengths of geodesic paths joining $a$ and $b$. This distance is invariant under $G$. Moreover, for small enough neighborhoods this distance is equal to the length of the geodesic joining $x$ and $y$ since in such neighborhoods geodesics furnish a proper absolute minimum for the lengths of paths.

We shall use the symbol $S(x, r), x$ a point of $M$ and $r$ a positive real number, to designate the set of points of $M$ whose distance from $x$ is at most $r$, and we shall call that set the sphere about $x$ of radius $r$. The boundary of this sphere will be the set of points for which the distance is precisely $r$, the inside (or interior) will be the set for which the distance is less than $r$. We shall say that a sphere is convex if each pair of its points are the end points of a geodesic every inner point of which is inside the sphere. It will be worth keeping in mind that, on our definition, when a geodesic belongs to a convex sphere only its end points can belong to the boundary of the sphere.

It will be convenient to use a number of simple lemmas and we now turn to them.

LEMma 1. If $x$ is any point of $M$ there exists a neighborhood $U$ of $x$ such that if $a, b$, and $c$ are three distinct points of $U$ which lie on one geodesic in that order, then

$$
d(b, x)<\max [d(a, x), d(c, x)] .
$$

Let $r$ be a positive number such that every sphere of center $x$ and radius $r^{\prime} \leqq r$ is convex. ${ }^{3}$ Let $U$ be the interior of $S(x, r)$. If $a, b$, and $c$ are in $U$, consider the sphere $S\left(x, r_{1}\right)$ where $r_{1}=d(x, b)$. The boundary of this sphere meets the geodesic $a b c$ at $b$ and this means that either $a$ or $c$ is outside $S\left(x, r_{1}\right)$ as otherwise the fact of convexity of $S\left(x, r_{1}\right)$ would be contradicted. But if a point is outside $S\left(x, r_{1}\right)$ its distance from $x$ is greater than $r$, and this concludes the proof.

LEMmA 2. If $x$ is any point of $M$, there exists a neighborhood $U$ of $x$ such that if $a, b, c$, and $e$ are four distinct points in $U$ and $a, b$, and $c$ are on a geodesic in that order then

$$
d(b, e)<\max [d(a, e), d(c, e)] .
$$

Here we shall let $U$ designate the interior of $S(x, r / 2)$ where $r$ has the same meaning as in the preceding lemma. Since $G$ is transitive over $M$ there will be an element $g$ in $G$ such that $g(e)=x$. The points

${ }^{3}$ For the existence of such a number $r$ in a Riemann space see the article by Whitehead, Convex regions in the geometry of paths, Quarterly Journal of Mathematics, vol. 3 (1932), pp. 33-42. 
$g(a), g(b)$, and $g(c)$ are inside $S(x, r)$, and they are on a geodesic since the isometry $g$ preserves geodesics. Making use of Lemma 1, and using again the fact the $g$ is an isometry, we complete the proof of this lemma.

Lemma 3. If $x$ is a point of $M$ there is a neighborhood $U$ of $x$ such that if $a, b, c$ are distinct points lying in that order on a geodesic in $U$ and if $d, e, f$ are distinct and lie in that order on a geodesic in $U$ then

$$
d(b, e)<\max [d(a, d), d(a, f), d(c, d), d(c, f)] .
$$

First we conclude from Lemma 2 that $d(b, e)$ is either less than $d(a, e)$ or less than $d(c, e)$. Say the notation is such that

$$
d(b, e)<d(a, e) .
$$

Now using Lemma 2 again we have

$$
d(a, e)<\max [d(a, d), d(a, b)],
$$

and Lemma 3 is proved.

LEMMA 4. If $x$ is any point in $M$, there exists a neighborhood $U$ of $x$ such that if $H$ is a closed subgroup of $G$ and $H(x)^{4}$ is in $U$ then $H$ has a fixed point in $U$.

Let $U$ denote some convex sphere about $x$ which satisfies the conclusion of Lemma 3. We shall show that this $U$ fulfills the conclusion of the present lemma. Suppose, therefore, that $H$ is a subgroup of $G$ such that $H(x)$ is contained in $\bar{U}$.

Let $q$ be a point in $\bar{U}$ such that $H(q)$ is of minimum diameter with respect to all orbits of $H$ which lie entirely in $\bar{U}$. If $H(q)=q$ our proof is completed so we may take it that $H(q) \neq q$.

In this case $H(q)$ contains at least two points. Let $b$ denote the midpoint of some geodesic determined by a pair of points of $H(q)$; now, because $H$ is a group of isometries, $H(b)$ is entirely included in the set of such midpoints, and $H(b)$ lies in $U$. It will now be shown that $H(b)$ has a smaller diameter than $H(q)$ and this will be a contradiction to our choice of $q$. This contradiction will complete the proof of our lemma.

The diameter of $H(b)$ coincides with the distance between two points of $H(b)$. This implies that there is an $h$ in $H$ such that the diameter of $H(b)$ is

$$
d[b, h(b)]
$$

\footnotetext{
${ }^{4}$ This is the orbit of the point $x$ under the group $H$.
} 
The point $b$ is the midpoint of a geodesic $a b c$ where $a$ and $c$ are in $H(q)$. The point $h(b)$ is on the geodesic $h(a), h(b), h(c)$. Calling these points $d, e$, and $f$ we may apply Lemma 3 and conclude that

$$
d[b, h(b)]<\max \{d[a, h(a)], d[a, h(c)], d[c, h(a)], d[c, h(c)]\} .
$$

This shows that the diameter of $H(b)$ is smaller than that of $H(q)$ and completes the proof of this lemma.

It is now a simple matter to conclude the proof of our theorem. We have to recall that the "points" of the space $M$ are the cosets $g G^{*}$, $g$ in $G$. Let us now interpret Lemma 4 in this light, with the group $G^{*}$ taken as the point $x$. The open set $U$ of the lemma corresponds to a system of cosets $g G^{*}$ and the set of elements of $G$ which belong to one of these cosets constitutes an open set $O$ of $G$. The set $O$ is a neighborhood of $G^{*}$.

Now let $H$ be a subgroup of $G$ which lies in $O$ and let $h$ be an element of $H$. Then, by the construction of $O$, every element of the coset $h G^{*}$ belongs to $O$ and the point $h G^{*}$ of $M$ belongs to $U$ in $M$. Therefore the premises of Lemma 4 are satisfied and we may suppose that $H$ has a fixed point in $M$.

This means that there is a "point" $g G^{*}$ of $M$ such that

$$
H\left(g G^{*}\right)=g G^{*} .
$$

This, however, is an equation in sets in $G$. We may write it

$$
H g G^{*}=g G^{*} .
$$

From this we conclude that

$$
g^{-1} H g G^{*}=G^{*} \text {. }
$$

But this means that for every choice of an element $k$ in $G^{*}$ and element $h$ in $H: g^{-1} h g k$ is contained in $G^{*}$.

This means that for every choice of $h$ in $H: g^{-1} h g$ is contained in $G^{*}$ and then finally, that the group $g^{-1} H g$ is a subgroup of $G^{*}$. This concludes the proof of our theorem.

COROLlARY. The element $g$ of Theorem 1 may be chosen within any prescribed neighborhood of the identity if the open set $O$ is chosen thereafter sufficiently small.

This corollary is a consequence of our method of proof. If the neighborhood $U$ of the last lemma is small $H$ will have a fixed point $y$ near $x$. Because of the transitivity of $G$ over $M$ there is a $g$ in $G$ such that $g=g(x)$ and this $g$ may be chosen near the identity in $G$ if $y$ is near enough to $x$. 
These results may be extended slightly in certain directions as follows :

TheOREM 2. Let $G$ be a compact connected finite-dimensional group and let $G^{*}$ be a closed connected subgroup. There exists an open set $O$ containing $G^{*}$ such that if $H$ is a closed connected group in $O$ then $H$ is the transform of a subgroup of $G^{*}$.

Let $Z$ be an invariant zero-dimensional subgroup of $G$ such that $f(G)=G / Z$ is a Lie group. By Theorem 1 there exists an open set $O_{1}$ containing $f\left(G^{*}\right)$ such that any subgroup of $f(G)$ which is in $O_{1}$ can be transformed into a subgroup of $f\left(G^{*}\right)$. Let $O=f^{-1}\left(O_{1}\right)$, and let $H$ be a closed connected subgroup of $G$ which is contained in $O$. There is a $g_{1}$ in $f(G)$ such that $g_{1}^{-1} f(H) g_{1}$ is in $f\left(G^{*}\right)$. Let $g$ be an element in $f^{-1}\left(g_{1}\right)$. Then $g^{-1} H g$ is a connected group such that $f\left(g^{-1} H g\right)$ is in $f\left(G^{*}\right)$. This means that every element of $g^{-1} \mathrm{Hg}$ belongs to the collection of elements $G^{*} Z=f^{-1} f\left(G^{*}\right)$.

The set of elements $G^{*} Z$ forms a group which must have the same dimension as $G^{*}$ for both are taken by $f$ into $f\left(G^{*}\right)$ and the homomorphism $f$, since it has a zero-dimensional kernel, preserves dimension. ${ }^{5}$ Hence the component of the identity of $G^{*} Z$ must be $G^{*}$. For in any case this component certainly includes $G^{*}$ and it cannot be larger than $G^{*}$, because if a finite-dimensional connected compact group contains another connected closed group as a proper subgroup it must be of higher dimension than this subgroup. This fact can be seen generally by observing that a similar fact is true for Lie groups.

If the connected group $g^{-1} H g$ is in $G^{*} Z$ it must be in the component of the identity of $G^{*} Z$. Therefore $g^{-1} H g$ is in $G^{*}$ as we wished to prove.

Smith College and

Queens College

${ }^{5}$ See van Kampen, Note on a theorem of Pontrjagin, American Journal of Mathematics, vol. 58 (1936), pp. 177-180, especially p. 178. 